\title{
Clinical use and evaluation of coded excitation in B-mode images
}

\author{
Misaridis, Athanasios; Pedersen, M. H.; Jensen, Jørgen Arendt
}

Published in:

Ultrasonics Symposium, 2000 IEEE

Link to article, DOI:

10.1109/ULTSYM.2000.921647

Publication date:

2000

Document Version

Publisher's PDF, also known as Version of record

Link back to DTU Orbit

Citation $(A P A)$ :

Misaridis, A., Pedersen, M. H., \& Jensen, J. A. (2000). Clinical use and evaluation of coded excitation in B-mode images. In Ultrasonics Symposium, 2000 IEEE (pp. 1689-1693). IEEE.

https://doi.org/10.1109/ULTSYM.2000.921647

\section{General rights}

Copyright and moral rights for the publications made accessible in the public portal are retained by the authors and/or other copyright owners and it is a condition of accessing publications that users recognise and abide by the legal requirements associated with these rights.

- Users may download and print one copy of any publication from the public portal for the purpose of private study or research.

- You may not further distribute the material or use it for any profit-making activity or commercial gain

- You may freely distribute the URL identifying the publication in the public portal 


\title{
Clinical use and evaluation of coded excitation in B-mode images
}

\author{
Thanassis X. Misaridis ${ }^{1}$, Morten H. Pedersen ${ }^{2}$ and Jørgen A. Jensen ${ }^{1}$ \\ ${ }^{1}$ Center for Fast Ultrasound Imaging, \\ Department of Information Technology, Bldg. 344, \\ Technical University of Denmark, DK-2800 Lyngby, Denmark \\ ${ }^{2}$ Herlev University Hospital, Department of Ultrasound, DK-2730 Herlev, Denmark
}

\section{Abstract}

Use of long encoded waveforms can be advantageous in ultrasound imaging, as long as the pulse compression mechanism ensures low range sidelobes and preserves both axial resolution and contrast. A coded excitation/compression scheme was previously presented by our group, which is based on a predistorted FM excitation and a mismatched compression filter designed for medical ultrasonic applications. The attenuation effect, analyzed in this paper using the ambiguity function and simulations, dictated the choice of the coded waveform. In this study clinical images, images of wire phantoms, and digital video demonstrate the applicability, clinical relevance, and improvement attained with the proposed scheme.

A commercial scanner (B-K Medical 3535) was modified and interfaced to a software configurable transmitter board and to a sampling system with a 2 GB memory storage. The experimental system was programmed to allow alternating excitation on every second frame. That offers the possibility of direct comparison of the same set of image pairs; one with pulsed and one with encoded excitation. Abdominal clinical images from healthy volunteers were acquired and statistically analyzed by means of the auto-covariance matrix of the image data. The resolution laterally is retained, axially is improved, while there is a clear increase in penetration.

Phantom images using the proposed FM-based scheme as well as complementary Golay codes were also acquired, in order to quantitatively evaluate the characteristics of the compressed output and its stability to attenuation. Images of a wire phantom in water show that the range sidelobes resulting from pulse compression are below the acoustic noise, which was at $50 \mathrm{~dB}$ for the pulsed images. For images acquired from an attenuation phantom, the proposed compression scheme was robust to frequency shifts resulting from attenuation. The range resolution is improved $12 \%$ by the coded scheme compared to a 2-cycle pulse excitation. For the maximum acquisition depth of $15 \mathrm{~cm}$, where the coded excitations are primarily intended, the improvement in SNR was more than $10 \mathrm{~dB}$, while the resolution was retained.

\section{Introduction}

Application of coded excitation in medical ultrasound has been scarce in the literature [1],[2]. Most of these attempts have not paid much attention to the choice of coded waveform apart from its autocorrelation properties. However, in the highly attenuating scattering medium of the human body, it will be shown that the matched filter output is not the autocorrelation function, and attenuation should be taken into account in the coded signal design.

\section{Modulated signals in attenuating media}

Let $s(t)=\alpha(t) \cdot \cos \left[2 \pi f_{0} t+\phi(t)\right]$ be a real modulated signal transmitted by the ultrasound transducer, where $f_{0}$ is the center frequency of the transducer and $\alpha(t), \phi(t)$ are the amplitude and phase modulation functions, respectively. If $s(t)$ is a narrowband signal, the complex representation $\psi(t)$ of $s(t)$ is analytical and can be written as

$$
\psi(t)=\mu(t) \cdot e^{j 2 \pi f_{0} t},
$$

where $\mu(t)$, often called the complex envelope of the signal, combines amplitude and phase modulation:

$$
\mu(t)=|\mu(t)| \cdot e^{j \phi(t)} .
$$

The real signal becomes:

$$
s(t)=\operatorname{Re}\{\psi(t)\}=|\mu(t)| \cos \left[2 \pi f_{0} t+\phi(t)\right],
$$

When an ultrasound transducer is excited with such a modulated signal, the spectrum is weighted by the transducer bellshaped spectrum. For a linear FM signal with a rectangular spectrum amplitude, the shape of the transmitted pulse spectrum amplitude is effectively that of the transducer. When the medium has no attenuation, the returned signal from a single scatterer is simply a time-shifted version of the transmitted signal:

$$
r(t)=\psi\left(t-\tau_{0}\right)=\mu\left(t-\tau_{0}\right) \cdot e^{j 2 \pi f_{0}\left(t-\tau_{0}\right)}
$$


where $\tau_{0}$ is the time instant after the start of transmission $(t=$ 0 ), at which the signal is being received.

The frequency dependence of the attenuation results in a larger attenuation of high frequencies than lower, when this signal propagates through the tissue. That will decrease the upper part of the bandwidth, causing effectively a decrease in the mean frequency that is a linear function of depth for a Gaussian-shaped pulse [3]. A significant downshift can be seen even for moderate tissue depths. For a transducer with a center frequency of $4 \mathrm{MHz}$ and a $65 \%$ fractional bandwidth, there is a downshift of $800 \mathrm{KHz}$ at a depth of $16 \mathrm{~cm}$. For moving targets, there is an additional Doppler shift, which is, however, two orders of magnitude less than the frequency downshift due to attenuation.

Assuming that attenuation does not distort the complex envelope of the modulated signal, and it only causes a frequency downshift $f_{d}$, the returned signal in an attenuating medium will be

$$
\begin{aligned}
r(t) & \simeq \mu\left(t-\tau_{0}\right) \cdot e^{j 2 \pi\left[\left(f_{0}-f_{d}\right)\left(t-\tau_{0}\right)\right]} \\
& \simeq \mu\left(t-\tau_{0}\right) \cdot e^{j 2 \pi\left[f_{0}\left(t-\tau_{0}\right)-f_{d}\left(t-\tau_{0}\right)\right]}
\end{aligned}
$$

\section{Matched Filter Processing and the Ambiguity Function}

The filter that maximizes the peak signal-to-noise ratio in the presence of white noise is the matched filter. The transfer function of the matched filter is the complex conjugate of the signal spectrum. Matched filtering of the received signal given in (5) requires a priori knowledge of the unknown parameters $\tau_{0}$ and $f_{d}$. Practically there is no need to match to the target delay $\tau_{0}$, since a change in delay merely changes the time at which the output occurs.

In the general case, the returned signal is matched to the signal $\psi(t-\tau) \cdot e^{j 2 \pi f_{m}(t-\tau)}$ with parameters $f_{m}$ and $\tau$. This corresponds to a filter with an impulse response of

$$
h_{\tau, f_{m}}(t)=\psi^{*}(T-t+\tau) \cdot e^{-j 2 \pi f_{m}(T-t+\tau)}
$$

The receiver's output at time $T$ (translated so that the maximum occurs at $\tau=0$ ) will then be

$$
\Psi_{\text {out }}(\tau)=e^{j 2 \pi f_{0} \tau_{0}} \cdot \chi\left(\tau-\tau_{0}, f_{m}-f_{d}\right)
$$

where $\chi\left(\tau, f_{d}\right)$ is defined as the ambiguity function, and is given by

$$
\begin{aligned}
\chi\left(\tau, f_{d}\right) & =\int_{-\infty}^{\infty} \psi(t) \cdot \psi^{*}(t-\tau) \cdot e^{j 2 \pi f_{d} t} d t \\
& =\int_{-\infty}^{\infty} \mu(t) \cdot \mu^{*}(t-\tau) \cdot e^{j 2 \pi f_{d} t} d t
\end{aligned}
$$

The ambiguity function (AF) is the complete 2-D matchedfilter response in scatterer range and frequency downshift. In the absence of attenuation (or generally when $f_{m}-f_{d}=0$ ), the matched filter output is the autocorrelation function of the waveform.

The squared modulus of the ambiguity function is referred to as the ambiguity surface (AS) [4]. The volume under the AS indicates the total interference contributed by each target, and the distribution of the volume is the distribution of this interference in range and frequency shift [4]. The volume under the AS is only a function of the signal's energy and cannot be reduced by any modulation. Coding simply redistributes this volume on the $\tau-f_{d}$ plane

\section{Implications of attenuation on signal design}

Tissue attenuation in ultrasound causes a significant frequency shift of the received signal. For coded excitation and some kind of matched filtering on the receiver, the compressed output is not the autocorrelation function of the code, but a cut of the ambiguity function for a certain frequency downshift. Therefore the AF of the transmitted waveform ought to have desired properties in the entire delayfrequency shift plane. One solution is to use a filter bank of $i$

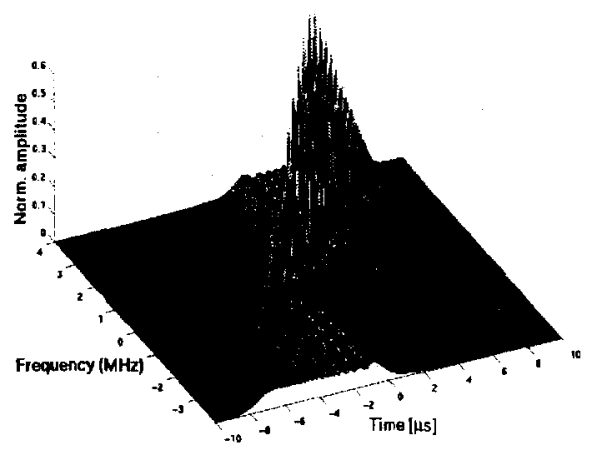

Figure 1: Ambiguity function of the linear FM waveform.

depth-dependent filters with impulse responses $h_{\tau, f_{m_{i}}}$, so that $f_{m_{i}}=f_{d_{i}}$, where $f_{d_{i}}$ is the estimated frequency shift at a certain depth. However, depending on the interrogating medium, there will always be deviations from the estimated values, and the requirement for robustness of compression in frequency shifts remains.

If attenuation is not compensated for, the ambiguity function should not have increased sidelobes off the delay axis. Figure 1 shows the ambiguity function of the proposed linear FM waveform. The advantage of this signal is that compression still occurs for a frequency mismatch. This occurs because its group delay is a linear function of frequency, and thus, the effect of frequency shift is a simple time translation of the compressed pulse. Note that a coded waveform with a nonlinear phase function (for example a non-linear FM waveform) will not be compressed, when there is a frequency shift. 
Most binary phase codes share the undesirable property of being quite sensitive to frequency shifts. The AF of such signals will generally have a central spike on a wide pedestal of range-frequency sidelobes. A search for binary codes with good autocorrelation functions is quite useless in ultrasound, if the ambiguity function away from the delay axis is unsuitable [4]. The Barker codes, for instance, do not retain their "perfect" compression output even with moderate frequency shifts. Binary phase codes insensitive to frequency shifts do exist, as for instance the Frank polyphase code, which is a discrete approximation of contiguous linear FM segments. Thus, the shape of the ambiguity function should be an additional design tool for search of such codes.

Complementary codes consist of two or more sequences of equal length, whose autocorrelation functions have sidelobes
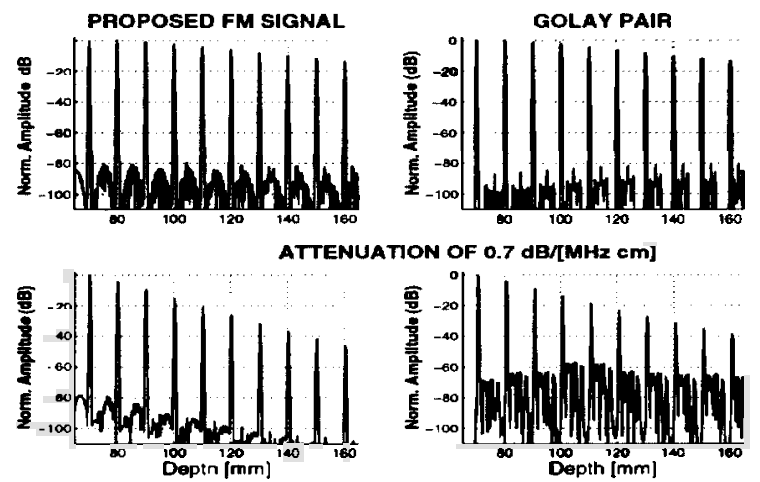

Figure 2: The effect of attenuation in the complementarity properties of Golay codes. Simulation results show a comparison of the echoes from 10 point scatterers between the proposed FM-based compression scheme and a Golay pair. The upper part is when there is no attenuation in the medium.

equal in magnitude but with inverse sign. Adding the autocorrelations of such pairs cancels the sidelobes entirely and doubles the mainlobe. The most well know complementary codes are the Golay codes. Simulation results in Fig. 2 show a comparison of the echoes from 10 point scatterers for the proposed FM-based compression scheme and a Golay pair. These plots show how these coded waveforms are affected by attenuation. While perfect sidelobe cancellation occurs for the complementary Golay codes, when the medium has no attenuation (upper plots), the complementarity property degrades with attenuation. This can be assessed by inspecting the ambiguity function of Golay codes. With an attenuation of $0.7 \mathrm{~dB} /[\mathrm{MHz} \times \mathrm{cm}]$, the range sidelobes increase up to -25 $\mathrm{dB}$ at a depth of $16 \mathrm{~cm}$, in contrast to the proposed tapered FM signal, whose compression is very robust to attenuation.

Based on these observations, the linear FM waveform seems the appropriate coded waveform for the highly attenuating medium of medical ultrasound, as long as the pulse compression mechanism ensures low range sidelobes and good axial resolution. Techniques for linear FM signal predistortion and mismatched filtering have been presented previously by our group [5]. In this paper, we evaluate the performance of these techniques experimentally. Images with complementary phase codes are also evaluated in an attenuating phantom.

\section{Materials and Methods}

\section{Measurement System}

An experimental digital coded excitation system was constructed (Fig. 3). A commercial scanner (B-K Medical 3535) was interfaced to one transmitter and one receiver board from the Center's newly constructed RASMUS experimental system [6]. A single-element mechanically rotating $4 \mathrm{MHz}$

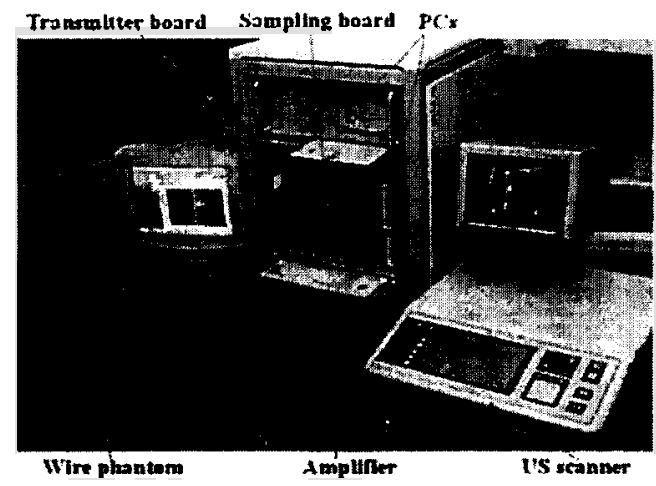

Figure 3: The experimental system.

probe (B-K Medical 8536) was used. The software control of both boards is implemented as a MATLAB ${ }^{T M}$ toolbox of high-level commands. The transmitter board is capable of transmitting different complex arbitrary waveforms for each line of an image with a few lines of MATLAB ${ }^{T M}$ code. The board was programmed to allow alternating excitation on every second frame. That allowed direct comparison of the same set of image pairs; one with conventional and one with encoded excitation, or image pairs with complementary sequences. The analog RF data of the scanner were sampled at $40 \mathrm{MHz}$. The receiver sampling unit has 12-bit ADCs and 2 GBytes storage SDRAM. That allowed storage of 140 consecutive ultrasound images, corresponding to about $25 \mathrm{~s}$ of scan data. Subsequently the stored RF data were read from the memory, and all post processing (pulse compression, interpolation, scan conversion and display) was done off-line on the computer. 


\section{Performance evaluation}

The performance of a pulse compression system can be measured using the four following indices:

i) The range mainlobe width, a measure of the range resolution.

ii) Range sidelobe measures, a measure of the induced "self-noise". Frequently used indices are the peak sidelobe level (PSL) and the integrated sidelobe level (ISL). PSL is simply the ratio between the highest sidelobe and the mainlobe. ISL is the the ratio of the total sidelobe energy to main peak energy expressed in decibels.

iii) The gain in signal-to-noise ratio (GSNR).

iv) Robustness to attenuation. There is no quantitative index reported. The shape of the ambiguity function can be used as a qualitative tool.

We have reported a compression scheme using a predistorted linear FM waveform and weighted mismatched filtering that has excellent measures of these indices [5]. The compressed output from this scheme has a theoretical $-20 \mathrm{~dB}$ mainlobe width of $1.48 \lambda$, very low axial sidelobes (PSL $=88 \mathrm{~dB}$ ) and $\mathrm{GSNR}=10.5 \mathrm{~dB}$. Figure 1 shows the robustness of this waveform to ultrasound attenuation.
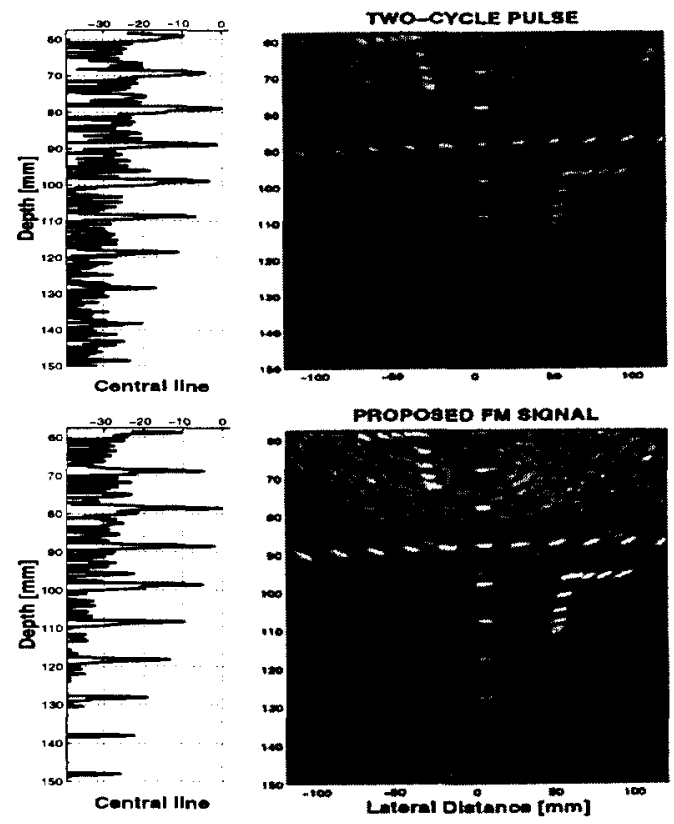

Figure 4: Detail of images of a wire phantom (right) and central RF lines (left) for coded and pulsed excitation. Matched filtering has been applied to both images. The dynamic range of the images is $45 \mathrm{~dB}$. There is an improvement in SNR of more than $10 \mathrm{~dB}$. Axial resolution is also higher for the coded image.

\section{Experimental results}

Initially, pulse-echo measurements from a wire phantom in water were performed. It has not been possible to evaluate the sidelobe level of the compressed output for the proposed code, but we can conclude that the sidelobes are below -50 $\mathrm{dB}$, which was the acoustic noise for the pulsed excitation output. The acoustic noise was due to reverberation at the water surface and the bottom of the tank. The $-6 \mathrm{~dB}$ axial resolutions were measured and were found to be $0.94 \lambda$ for the coded and $1.18 \lambda$ for a two-cycle pulsed excitation.

Images of an attenuating wire phantom are shown in Fig. 4 and 5. The power levels of the excitation signals on the trans-
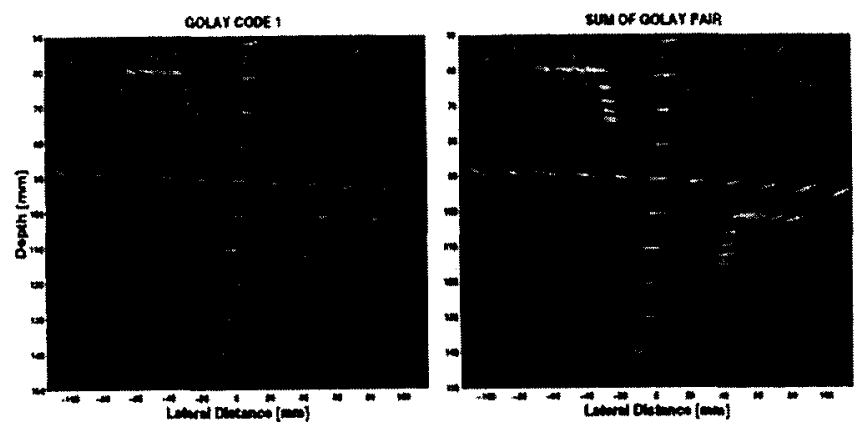

Figure 5: Images with Golay pair excitation of a wire phantom with attenuation of $0.5 \mathrm{~dB} /[\mathrm{MHz} \times \mathrm{cm}]$. On the left is the image with one of the Golay codes and on the right is the sum of the two complementary images. Dynamic range $=45 \mathrm{~dB}$.

ducer were lower than the original excitation power of the scanner. The phantom has attenuation of $0.5 \mathrm{~dB} /[\mathrm{MHz} \times \mathrm{cm}]$ and consists of wires of $0.2 \mathrm{~mm}$ in in diameter, positioned every $1 \mathrm{~cm}$ axially. Additional wires are placed at a 15 degree angle with decreasing distance down to $1 \mathrm{~mm}$. In the pulsed image of Fig. 4 a two-cycle pulse of the carrier frequency was used. In the coded image the excitation was a $20 \mu$ s tapered FM signal with the compression filter being the one from [5]. The $-6 \mathrm{~dB}$ axial resolution of the coded image measured at the wires in depth 12 and $14 \mathrm{~cm}$ was $1.31 \lambda$. The resolution of the pulsed image was $1.49 \lambda$. In conventional imaging, short broadband pulses have to be used in order to achieve good axial resolution and to use all available system bandwidth, with the drawback of degrading the SNR. When FM signals are transmitted, the axial resolution depend on the bandwidth that is swept and not on the duration of the signal. Therefore, coded images using FM modulated signals result not only in a better SNR, but also in better axial resolution by utilizing the available bandwidth more efficiently. Fig. 4 shows that there is an effective gain in SNR of $10 \mathrm{~dB}$ or more, that corresponds to an additional penetration of $3-4 \mathrm{~cm}$ with the $4 \mathrm{MHz}$ probe.

Images of the same phantom using a complementary pair 
of Golay codes with length 40 are shown in Fig. 5. A single Golay code has high axial sidelobes that are visible on the left image. When the echoes from 2 Golay codes are added
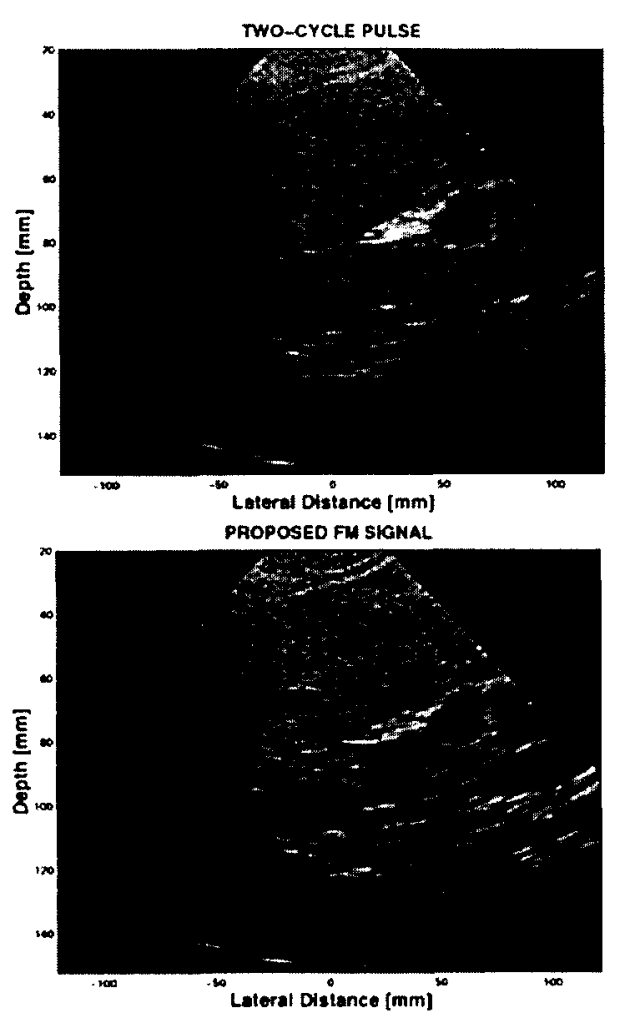

Figure 6: Clinical images of the right kidney for coded and pulsed excitation. The portal vein and the inferior vena cava are at the right side of the images and liver tissue is left from the kidney. The dynamic range of the images is $45 \mathrm{~dB}$. Improvement in resolution and noise reduction at large depths are visible.

coherently, there is a degree of cancellation on the axial sidelobes. However, confirming our hypothesis, the cancellation of the sidelobes is not perfect due to attenuation, and shadows are still visible along the wires.

Clinical images of the abdomen using the proposed scheme are shown in Fig. 6. The images show an excellent performance of the encoded excitation in terms of noise reduction at large depths and resolution. The auto-covariance matrix on the image gives an indication of the speckle size. The lateral resolution of speckle data from the images of Fig. 6 is very similar for both images, while axially the coded image has slightly better performance (Fig. 7).

These results clearly demonstrate that abdominal ultrasound imaging can benefit from coded excitation yielding a higher SNR and therefore penetration, while maintaining both axial and lateral resolution. The higher SNR can be exchanged with resolution by increasing the center frequency, i.e. for $\mathrm{GSNR}=10 \mathrm{~dB}$, going from 4 to $5 \mathrm{MHz}$ without compromising SNR. Longer codes can make this frequency step even bigger.
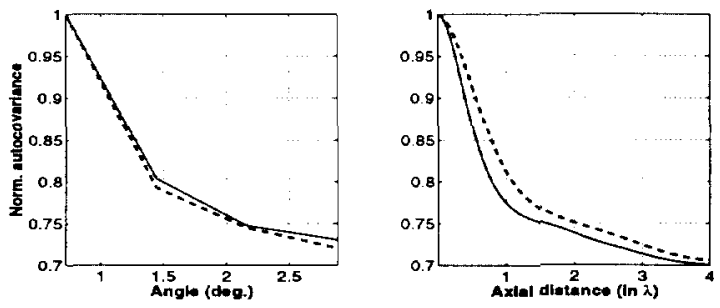

Figure 7: Lateral and axial resolution in speckle using autocovariance matrix analysis. The dashed lines are for the pulsed image.

\section{Acknowledgments}

The authors would like to thank Ph.D. student B. Tomov for his extensive work on the experimental system and Dr. P. Munk for valuable discussions. This work was supported by grant 9700883 and 9700563 from the Danish Science Foundation and by B-K Medical A/S.

\section{References}

[1] M. O'Donnell. Coded excitation system for improving the penetration of real-time phased-array imaging systems. IEEE Trans. Ultrason., Ferroelec., Freq. Contr., 39:341-351, 1992.

[2] J. Shen and E. S. Ebbini. A new coded-excitation Ultrasound imaging system - Part I: Basic principles. IEEE Trans. Ultrason., Ferroelec., Freq. Contr., 43:131-140, 1996.

[3] J. A. Jensen. Estimation of Blood Velocities Using Ultrasound: A Signal Processing Approach. Cambridge University Press, New York, 1996.

[4] A. W. Rihaczek. Principles of high-resolution radar. McGraw-Hill, New York, 1969.

[5] T. X. Misaridis and J. A. Jensen. An effective coded excitation scheme based on a predistorted FM signal and an optimized digital filter. In Proc. IEEE Ultrason. Symp., volume 2, pages 1589-1593, 1999.

[6] J. A. Jensen, O. Holm, L. J. Jensen, H. Bendsen, H. M. Pedersen, K. Salomonsen, J. Hansen, and S. Nikoloy. Experimental ultrasound system for real-time synthetic imaging. In Proc. IEEE Ultrason. Symp., volume 2, pages 1595-1599, 1999. 